\title{
Electrical Current
}

National Cancer Institute

\section{Source}

National Cancer Institute. Electrical Current. NCI Thesaurus. Code C48434.

The movement of electrical charges in a conductor. 